\title{
Production and Optimization of Polyhydroxybutyrate from Rhizobium sp. present in root nodules
}

\author{
${ }^{1}$ R.Sita Lakshmi, ${ }^{2}$ Hema.T. A., ${ }^{3}$ Divya .T. Raj, ${ }^{4}$ Starin Shylaja .T. \\ 1,2,3,4, Department of Microbiology, Malankara Catholic College, Mariagiri, Kaliakkavilai, 629 153, Tamil \\ Nadu.
}

\begin{abstract}
Bioplastics, a form of plastics derived from renewable biomass such as vegetable oil, corn starch, pea starch or microbiota. The biopolymer polyhydroxybutyrate, polyester produced by certain bacteria during the addition of excess carbon sources. In the present study a gram negative PHB accumulating microbe was isolated and identified. Based on the morphological and physiological properties it was identified as Rhizobium species. The different media parameter such as $\mathrm{pH}$, carbon sources, nitrogen sources and temperature was optimized for increased PHB accumulation. Based on different $p H, p H 5$ was more suitable for increased PHB accumulation by Rhizobium. According to different carbon and nitrogen sources, glucose and potassium nitrate showed high PHB accumulation. Based on the effect of different temperature $30^{\circ} \mathrm{C}$ was more suitable for increased amount of PHB accumulation. PHB production by this strain was in the stationary phase of their growth. PHB was extracted by chloroform and the extracted polymer was determined by UV-VIS spectrophotometer at $235 \mathrm{~nm}$.
\end{abstract}

Keywords: Polymer, polyhydroxybutyrate, Rhizobium sp., temperature, UV-VIS spectrophotometer.

\section{Introduction}

Bioplastics, a special type of biomaterials derived from plant sources such as soybean oil, hemp oil or corn starch or microbial source, rather than traditional petrochemical derived plants. Microbial bioplastics were polyesters that are produced by a range of microorganism cultivated under various growth and nutrient conditions. These polymers usually lipids, accumulated as storage materials (as mobile, amorphous, liquid granules).

Bioplastics were made from a compound called poly hydroxyalkanoate (PHA). Bacteria accumulating PHA in the presence of excess carbon source, is similar to how humans accumulated fat deposits on their body. Poly-3 - hydroxybutyric acid is the most common microbial polyhydroxyalkanoate. Polyhydroxybutyrate accumulated as energy reserve material in many micro-organisms like Alcaligenes, Bacillus, Nocardia, Pseudomonas, Rhizobium etc. Alcaligenes eutrophus and Azotobacter beijerinickii can accumulate upto $70 \%$ of their dry weight of PHB. These microorganisms could produce the polymer in environment of nitrogen and phosphorous limitation. PHB had implicated as an energy source in the symbiotic nitrogen fixation process between the bacterial genera Rhizobium, Bradyrhizobium and leguminous plants ( Karr et al., 1984). Root nodules are produced by these bacteria and microorganisms exist as bacteroids. The heavy demand for energy for nitrogen fixation was met by the metabolism of photosynthetic carbon compounds (principally organic acids) transported to the root nodules. The microaerophilic conditions in the root nodule inhibited the TCA cycle enzymes such as citrate synthesis, NADP isocitrate dehydrogenase and 2-oxoglutarate dehydrogenase (Chandrasekharan et al., 1976) .

PHB is a compact right-handed helix with a two fold screw axis and a fiber repeat of $0.596 \mathrm{~m}$ (Cornibert et al., 1972). PHB was optically active, with the chiral centre of the monomer unit always in the R absolute configuration D-(-) in the traditional nomenclature. Their molecular weight can be up to 2 million (i.e. 20000 monomers per polymer molecules). PHB is water insoluble and relatively resistant to hydrolytic degradation. This differentiates PHB from most other currently available biodegradable plastics, which are either soluble or moisture sensitive. PHB had good oxygen permeability and good ultra-violet resistance but has poor resistance to acids and bases. PHB was soluble in chloroform and other chlorinated hydrocarbons. It was biocompatible and hence was suitable for medical applications. PHB had melting point $175^{\circ} \mathrm{C}$., and glass transition temperature $15^{\circ} \mathrm{c}$ (Senior et al., 1972). It had tensile strength $40 \mathrm{MPa}$ which is close to that of polypropylene. PHB sinks in water while polypropylene floats. But sinking of PHB facilitated its anaerobic biodegradation in sediments, it was nontoxic.

The average number of granules per cell remained constant at $12.7 \pm 1.0$ and $8.6 \pm 0.6$, respectively, and increased uniformly in average diameter from 0.24 to $0.5 \mu \mathrm{m}$ to accommodate the PHB produced. The PHB granules possess a membrane coat about $2 \mathrm{~nm}$ thick, composed of $0.5 \%$ lipid and $2 \%$ protein respectively of the granule weight (Lundgren et al., 1964). The activities of the PHB synthases and depolymerase are associated with this membrane protein. 
In vivo the PHB granules existed as crystalline structure. Treatment of native PHB granules with aqueous acetone to remove the membrane coat disclosed a fiber structure and long, parallel flexible fibers that coalesced to form ribbons which in turn, yielded the characteristic lath-shaped crystals of PHB. To degrade the polymer, the depolymerase enzyme must gain access to its crystalline substrate.

\section{Experimental, Materials and methods}

\section{Sample collection:-}

Healthy, pink and undamaged root nodules of Phaseolous vulgaris leguminous plant were collected in a sterile polythene bags and brought to the laboratory Department of Microbiology, Malankara Catholic College, Mariagiri.

\section{Isolation of Rhizobium:-}

The Phaseolous vulgaris leguminous plant was carefully uprooted from the soil and the root system was washed with running water to remove the soil particles firm, undamaged, pink coloured root nodules were selected from the roots. The root nodules were dipped in $0.1 \%$ acidified mercuric chloride solution for 5 minutes to sterilize the surface of the nodules. The sterilized root nodules were then washed five or six times with distilled water. The root nodules were once again sterilized by immersing them in $90 \%$ ethyl alcohol for 10 seconds and washed repeatedly with distilled water. The root nodules were crushed gently in a small amount of distilled water using a mortar and pestle to get a suspension. The suspension was diluted and inoculated on to the Yeast Extract Mannitol agar medium (Himedia) in petri dishes. The culture plates were incubated at $37^{\circ} \mathrm{C}$ for about 10 days. Rhizobial cells form gummy colonies on the medium. The isolated cultures were maintained on to the yeast extract mannitol broth (Himedia) for further analysis (Cappucino and Sherman, 2004).

\section{Identification of Bacterial Isolates:}

The isolated colonies were identified using Bergey's Manual of Determinative Bacteriology (Holt et al., 1993) based on the characters such as morphology, physiology and nutritional, cultural characteristics and biochemical tests.

\section{PHB Staining}

A thin bacterial smear was made on the clean glass slide. The smear was air dried and stained it with Sudan black for 10 minutes. The smear was washed and air dried. Then stained it with safranin for 10 seconds and observed under oil immersion lens (Hartman, 1940).

\section{Assay of polyhydroxybutyrate:-}

Bacteria were grown on the yeast extract mannitol broth (Himedia) at $37^{\circ} \mathrm{C}$ for 48 hours on a shaker. A suspension of culture was centrifuged at $8000 \mathrm{x}$ g for 15 minutes. The supernatant was discarded and the pellet was treated with $10 \mathrm{ml}$ of sodium hypochlorite (Loba) and the mixture was incubated at $30^{\circ} \mathrm{C}$ for $2 \mathrm{hrs}$. After incubation, the mixture was centrifuged at $5000 \mathrm{x}$ g for 15 minutes and then washed with distilled water, acetone and methanol and extracted.

After washing, the pellet was dissolved in $5 \mathrm{ml}$ of boiling chloroform (Loba) and filtered through Whatman No.1 filter paper. The chloroform extract was evaporated to dryness (Law and Slepecky, 1961). Determination of PHB yield was performed by dry weight estimation. The ultraviolet absorption spectrum of the polymer was analysed following its conversion to crotonic acid by treatment with concenterated sulphuric acid and the absorbance was scanned at $235 \mathrm{~nm}$ with UV-VIS spectrophotometer.

\section{Estimation of cell dry weight:-}

After centrifugation of the culture medium, the supernatant was discarded and the pellet was washed with distilled water. The washed pellet was resuspended in $1 \mathrm{ml}$ distilled water, transferred to preweighed plates and dried to constant weight at $60^{\circ} \mathrm{C}$.

\section{Optimization of Nutritional parameters:-}

\section{Effect of different $\mathrm{pH}$ on PHB production:-}

The yeast extract mannitol broth was prepared at different $\mathrm{pH}$ such as 5, 6, 7, 8 and 9 by using $2 \mathrm{~N} \mathrm{Hcl}$ or $\mathrm{NaOH}$. The organism was inoculated into the broth and determined the optimum $\mathrm{pH}$ for the production of PHB by the microorganism.

\section{Effect of different carbon sources on PHB production:-}

$1 \%$ glucose, sucrose and arabinose as the carbon sources were added to yeast extract mannitol broth The organism was inoculated into the broth and determined the definite carbon source for the production of PHB by the microorganism. 
Production and Optimization of Polyhydroxybutyrate from Rhizobium sp. present in root nodules

\section{Effect of different nitrogen sources on PHB production:-}

Peptone and tryptone in the yeast extract mannitol broth was replaced by $1 \% \mathrm{~L}$ -

Cysteine and L-Glycine and potassium nitrate as nitrogen sources. The organism was inoculated into the broth and determined the optimum nitrogen source for the production of PHB by the microorganism.

\section{Effect of different temperature on PHB production:-}

The microorganism was inoculated into yeast extract mannitol broth and incubated at different temperature such as $30^{\circ} \mathrm{C}$ and $37^{\circ} \mathrm{C}$ on shaker at $100 \mathrm{rpm}$.

\section{Statistical analysis:-}

The correlation between production of PHB and dry cell weight was determined by spearman's correlation coefficient test (Conover, 1971).

\section{Results:}

The present study was carried out to isolate PHB producing microorganisms from the root nodule sample. The PHB production efficiency of isolated microorganisms was purified by repeated streaking on yeast extract mannitol agar plates. Based on morphological, physiological and biochemical characteristics the isolated and purified bacterial isolate was identified as Rhizobium sp. (Table. I). PHB production by this strain was in the stationary phase of their growth and it accumulated $0.34 \mathrm{~g} / \mathrm{l}$ PHB with $12.5 \%$ yield under stationary phase.

Table I. Morphological, physiological and Biochemical identification of bacterial isolate from root nodules. The isolate was identified as Rhizobium sp. according to Bergey's Manual of Systematic Bacteriology.

\begin{tabular}{|l|l|}
\hline \multicolumn{1}{|c|}{ Characteristics } & $\mathbf{R}_{\mathbf{1}}$ \\
\hline Gram staining & - \\
\hline Cell shape & rod \\
\hline Motility in liquid medium & + \\
\hline Flagellar arrangement & + or - \\
\hline PHB Staining & + \\
\hline Carbohydrate fermentation (glucose) & Acid \\
\hline Catalase test & + \\
\hline Oxidase test & + \\
\hline
\end{tabular}

$$
\begin{array}{lll}
\mathrm{R}_{1} \quad \text { - Root Nodule sample } & \text { + Positive }
\end{array}
$$

Typically, metabolic process was highly susceptible to even slight changes in $\mathrm{pH}$. Therefore, proper control of $\mathrm{pH}$ was critical. The experimental result showed that highest PHB content was obtained at $\mathrm{pH} 5$ (21.6\%) in Rhizobium (Table. II). Different carbon sources which affects PHB production was evaluated using various carbon sources (including glucose, sucrose and arabinose). Among this glucose supported maximum PHB production $(8 \%)$ in Rhizobium (Table.III). Nitrogen sources affecting PHB production was evaluated using various nitrogen sources including potassium nitrate, L-cysteine and L-Glycine. Among these tests in this study, potassium nitrate showed highest PHB content ( $8.6 \%$ ) in Rhizobium (Table.IV).

Table II. Statistical analysis represents the effect of pH on PHB Production and yield in Rhizobium sp.

\begin{tabular}{|l|l|l|l|l|}
\hline Sl.No & pH & Dry cell weight $(\mathbf{g} / \mathbf{l})$ & PHB & PHB yield (\%) \\
\hline 1 & 5 & $41 \pm 1.41$ & $1.88 \pm 0.014$ & 21.6 \\
\hline 2 & 6 & $52.5 \pm 0.707$ & $1.243 \pm 0.028$ & 12.5 \\
\hline 3 & 7 & $42.5 \pm 2.12$ & $1.360 \pm 0.028$ & 13.8 \\
\hline 4 & 8 & $54.5 \pm 3.535$ & $0.926 \pm 0.021$ & 0.92 \\
\hline 5 & 9 & $21 \pm 1.414$ & $0.847 \pm 0.02$ & 0.88 \\
\hline
\end{tabular}

Table III. Statistical analysis represents the effect of carbon sources on PHB Production and yield in Rhizobium sp.

\begin{tabular}{|l|l|l|l|l|}
\hline Sl.No & $\begin{array}{l}\text { Carbon } \\
\text { Sources }\end{array}$ & $\begin{array}{l}\text { Dry cell } \\
\text { weight(g/l) }\end{array}$ & PHB & $\begin{array}{l}\text { PHB yield } \\
(\%)\end{array}$ \\
\hline 1 & Glucose & $14.5 \pm 0.70$ & $0.75 \pm 0.70$ & 8 \\
\hline 2 & Sucrose & $6.5 \pm 0.707$ & $0.115 \pm 0.02$ & 1 \\
\hline 3 & Arabinose & $43.5 \pm 0.707$ & $0.32 \pm 0.00$ & 3 \\
\hline
\end{tabular}


Production and Optimization of Polyhydroxybutyrate from Rhizobium sp. present in root nodules Table IV. Statistical analysis represents the effect of nitrogen sources on PHB Production and yield in Rhizobium sp.

\begin{tabular}{|l|l|l|l|l|}
\hline Sl.No & Nitrogen Sources & Dry cell weight $(\mathrm{g} / \mathbf{l})$ & PHB & PHB yield (\%) \\
\hline 1 & L-glycine & $43 \pm 1.412$ & $0.401 \pm 0.015$ & 4.1 \\
\hline 2 & L-cysteine & $15 \pm 1.412$ & $0.679 \pm 0.975$ & 1.4 \\
\hline 3 & Potassium nitrate & $44.5 \pm 1.412$ & $0.401 \pm 0.015$ & 8.6 \\
\hline
\end{tabular}

Temperature, $\mathrm{pH}$ and agitation rate all affect dissolved oxygen levels and mass transfer efficiency, these environmental factors profoundly affect cellular growth and bioproduct production. The effect of temperature in PHB production showed highest accumulation at $30^{\circ} \mathrm{C}(25 \%)$ and an agitation rate of $100 \mathrm{rpm}$ under aerobic condition (Table V). The correlation between production of PHB and dry cell weight were determined by spearman's $\rho$ correlation coefficient test (Conover., 1971). In this work the media parameters were optimized with the dry cell weight and PHB production existed and the correlation was found $\rho=1$. When this value was compared with the table value, it was seen that the relationship was significant $(\rho=1)$.

Table V. Statistical analysis represents the effect of temperature on PHB Production and yield in Rhizobium sp.

\begin{tabular}{|c|c|c|c|c|}
\hline Sl.No & $\begin{array}{c}\text { Temperature } \\
\left({ }^{\mathbf{0}} \mathbf{C}\right)\end{array}$ & Dry cell weight(g/l) & PHB & $\begin{array}{c}\text { PHB yield } \\
(\mathbf{\%})\end{array}$ \\
\hline 1 & 30 & $42.25 \pm 1.76$ & $2.459 \pm 1.234$ & 25 \\
\hline 2 & 37 & $45.5 \pm 0.70$ & $0.170 \pm 1.321$ & 22 \\
\hline
\end{tabular}

\section{Discussion}

Rapid population growth in recent years had resulted in severe environment degradation. The conventional plastics were not degraded by microorganisms; many companies had attempted to develop biodegradable bioplastics. Bacteria able to synthesize PHA could be divided into two during the stationary phase and the second group during the growth phase. In "short chain length" PAH, such as PHB and PHV, carbon numbers of monomers are 3 to 5 . Polyhydroxybutyrate were the polyesters synthesized by microorganisms.

In the present study colonies were isolated from the root nodule sample by serial dilution and plating on yeast mannitol agar medium. The colonies were identified as PHB producing Rhizobium. This finding was supported by an earlier report said that most efficient PHB producing bacteria belong to the genus Rhizobium (Aslim. et al., 2002). The PHB production by this bacterium was confirmed by presence of PHB granules during Sudan black B staining. Based on different pH, pH 5 showed maximum amount of PHB (21.6\%). Tavernier et al., (1997) investigated the effects of different nitrogen and carbon sources and $\mathrm{pH}$ on exopolysaccharide (EPS) and PHB production in two strains of $R$. meliloti. They reported that these two strains showed different growth rates in the medium. They also noted that there was a decrease in PHB content in the medium with an acidic $\mathrm{pH}$. In the medium with fructose and yeast extract, the PHB yield was $85 \%$. According to the carbon sources, glucose showed maximum level of PHB content upto (8\%) in Rhizobium. The other study showed that sucrose contain maximum level of PHB content (Aslim .et al., 2002). But the result obtained in this study was contrary to the findings of (Aslim et al., 2002). By using potassium nitrate as nitrogen sources Rhizobium produced maximum level of PHB upto $(8.6 \%)$. The other study showed that L-cysteine and LGlycine highest content PHB content (Aslim et al., 2002). But the result obtained in this study was contrary to the findings of (Aslim et al., 2002). Bonartseva et al., (1994) tested the capacity for PHB production in active and less active strains of Rhizobium phaseoli, $R$. meliloti and $R$. trifolii during growth on media with different carbon and nitrogen sources. It was found that PHB synthesis can be selectively induced either in active or less active Rhizobium strains by sources of carbon and nitrogen. They reported that the less active strain of $R$. phaseoli 680 was a promising producer of PHB, and the PHB content in cells of this strain was up to $65 \%$ of dry cell weight during growth on a medium with sucrose and nitrate; the PHB content was much lower when organic acids were used. Based on the temperature Rhizobium produced increased PHB content at $30^{\circ} \mathrm{C}(25 \%)$. The correlation between cell dry weight and PHB content was significant relationship $(\rho=1)$. The identity and purity of the PHB obtained from Rhizobium had been confirmed by solubility properties and UV-absorption spectra. Digestion of the polymer with concentenated $\mathrm{H}_{2} \mathrm{SO}_{4}$ gave a sharp peak at $235 \mathrm{~nm}$ characteristic of crotonic acid indicating the presence of PHB. 
Production and Optimization of Polyhydroxybutyrate from Rhizobium sp. present in root nodules

Further optimization of the media and growth conditions with the best carbon and nitrogen sources as well as mutagenesis for further increase in PHB production by this strain will be performed.

\section{Literature}

[1] D.B. Karr, J.K. Waters, F. Suzuki and D.W. Emerich, Enzymes of the poly- $\beta$-hydroxybutyrate and citric acid cycles of Rhizobium japonicum bacteroids. Plant Physiol. 75, 1984, 1158-1162.

[2] P.T. Chandrasekharan and Y.I. Shethna, Purification and properties of an NADP ${ }^{+}$-specific isocitrate dehydrogenase from Rhizobium meliloti, J. Microbiol., 42, 1976, 471-482.

[3] J. Cornibert and Y.I. Marchessault, Physical properties of poly- $\beta$-hydroxybutyrate.III. Folding of helical segments in 2,2,2trifluoroethanol, Macromolecules, 3, 1972, 741-746.

[4] P.J. Senior, G.A. Beech, G.A.F. Ritchie and E.A. Dawes, The role of oxygen limitation in the formation of poly- $\beta$ hydroxybutyrate during batch and continuous culture of Azotobacter beijerinckii, Bioche. J., 128, 1972, $1193-1201$.

[5] D.G. Lundgren, R.M. Pfister and J.M. Merrick, Structure of poly ( $\beta$-hydroxybutyric acid) granules, J. Gen. Microbiol. 34, 1964, 441-446.

[6] Cappucino, Sherman, Microbiology A laboratory Manual (2004) $6^{\text {th }} \mathrm{ed}, 143-145,156-158,181-183$.

[7] J.G. Holt, N.R. Kreig, J.T. Sneath, J.T. Staley and S.T. William, Bergey's Manual of Determinative Bacteriolo,,. $9^{\text {th }}$ ed. 1993, $559-564$.

[8] T.L. Hartman, The use of Sudan Black B as a bacterial fat stain, Staining Technology, 15, 1940, $23-28$.

[9] W.J. Conover, Practical non parametric statistics (New York, JohnWiley and son., 1971) Inc. ISBN 0471168521.

[10] B. Aslim, Z . N. Yuksekdag and Y. Beyatli, Determination of PHB growth quantities of certain Bacillus species isolated from soil, Turk. E. J. Biotechnolo, 2002, 24-30.

[11] G. A . Bonartseva, V. L. Myshkina, E. D. Zagreba, Poly- $\beta$ - hydroxybutyrate content in cells of various Rhizobium species during growth with different carbon and nitrogen source,. Microbiol., 63(1), 1994, 45-48. 